\title{
Profile
}

\section{Collaborative Group on Drug Use in Pregnancy (CGDUP)}

Current findings from the WHO-sponsored Collaborative Group on Drug Use in Pregnancy were published in this Journal in 1991 (Vol. 2, No. 6). The Group is conceived as a permanent global network of investigators interested in determining the pattern of drug use, testing or validating therapeutic or prophylactic interventions, and examining other drug safety issues in mother and child. To promote and support further development of the network, a list of reference investigators provided below.

Bonati, Maurizio

Unità di Farmacologia Clinica Perinatale Istituto di Ricerche Farmacologiche

"Mario Negri"

Via Eritrea 62

20157 Milan, Italy

Tel. 0039-2-39014511

Fax 0039-2-33200049/3546277

\section{Study Coordinators}

Tognoni, Gianni

Laboratorio di Farmacologia Clinica Instituto di Ricerche Farmacologiche

"Mario Negri",

Via Eritrea 62

20157 Milan, Italy

Tel. 0039-20-39014511

Fax 0039-2-33200049/3546277

\section{Country Reference Investigators}

Bennett, Peter

School of Pharmacy and Pharmacology

University of Bath

Claverton Down

Bath BA2 7AY

United Kingdom

Tel. 0044-225-826826 Fax 0044-225-62508

Grant, Adrian

National Perinatal Epidemiology Unit

Radcliffe Infirmary

Oxford OX2 6HE

United Kingdom

Tel. 0044-865-224876 Fax 0044-865-792270
Blaakaer, Jan

Dept. of Gynaecology \& Obstetrics University Hospital (Rigshospitalet) Section 4031 Blegdamsvej 9 2100 Copenhagen

Denmark

Tel. 0045-38-41-211211

Culig, Josip

Dept. of Medicine

Rebro Clinical Hospital Centre

12, Kispaticeva

41000 Zagreb

Yugoslavia

Tel. 0048-41-211211 
Stanulovic, Milan

Medicinski Fakultet Novi Sad

OOUR Institut Predklinickih Disciplina

Zavodza Farmakologiju I Toksikologiju

Hajduk Veljkova 7-9 Post Pregradak 98

21001 Novi Sad-Yugoslavia

Tel. 0038-21-615771

De Jong-van den Berg, Lolkje

Dept. of Pharmacology and Pharmacotherapeutics

University of Groningen

Antonius Deusinglaan 2

9713 AW Groningen

The Netherlands

Tel. 0031-50-633272/633330 Fax 0031-50-633311

Romero, Marilena

Centro di Ricerche Farmacologiche e

Biomediche

Consorzio "Mario Negri" Sud

Via Nazionale

66030 S. Maria Imbaro (Chieti), Italy

Tel. 0039-0872-5701 Fax 0039-0872-578240

Diogene, Eduardo

Unitat de Farmacologia Clinica

Universitat Autonoma de Barcelona

Passeig de la Vall d'Hebron S/N

Barcelona 08035

Spain

Tel. 0034-3-4283029 Fax 0034-3-4283176

Do Carmo Batista Dias Dos Santos, Maria Hospital do Servidor Publico Estadual

Rua Diacui 37

São Paulo - Indianopolis

Brazil

Tel. 0055-11-5313952 Fax 0055-11-8726700

Harden, Hubert

Service Gynécologie-Obstétrique

Centre Hospitalier Princesse Grace

B.P. 480 Avenue Pasteur

98012 Monaco

Monaco

Tel. 0033-93-300818

Hemminki, Elina

Dept. of Public Health

University of Helsinki

Haartmaminkatu 3

Helsinki 00280

Finland

Tel. 00358-0-4346465 Fax 00358-0-434656
Hoffmann-Traeger, Annemarie

Institut für Klinische Pharmacologie

Universität Jena

Bachstrasse 18

Jena $0-6900$

Germany

Tel. 0037-78-132379 Fax 0037-78-822345

Kasilo, Ossy

Drug Information Toxicology Service - DATIS

Dept. of Pharmacology - Univ. of Zimbabwe

P.O. Box A 178

Avondale - Harare

Zimbabwe

Tel. 00263-4-790233 Fax 00263-4-732828

Nyazema, Norman

Dept. of Clinical Pharmacology

University of Zimbabwe

G. Huggins School of Med.

P.O. Box A 178

Avondale-Harare, Zimbabwe

Tel. 00263-4-791631 Fax 00263-4-30292

Kriska, Milan

Dept. of Pharmacology-Medicine Faculty

Comenius University

Sasinkova 4

81108 Bratislava

Czechoslovakia

Tel. 0042-7-62559

Mrazek, Milan

Research Institute for the Care of Mother and Child

Podoli, nabr. Marxe 157

14710 Prague 4

Czechoslovakia

Lee, David

Apartado 6-2873

Estafeta Dorado

Panama

Matheson, Ingrid

Dept. of Pharmacotherapeutics

Ullevâl University Hospital

University of Oslo

P.O. Box 1065

Blindern, N-OSLO 3 Norway

Tel. 0047-2-456050/56 Fax 0047-2-695401

Ofori-adjei, David

Centre for Tropical Clinical Pharmacology 
University of Ghana Medical School P.O. Box 4236

Accra

Ghana

Tel. 00233-21-665401 Fax 00233-21-668425

O'Herlihy, Colm

Dept. of Obstetrics and Gynaecology

National Maternity Hospital

Holles Street

Dublin 2

Ireland

Savona Ventura, Charles

Dept. of Obstetrics \& Gynaecology

St. Luke's Hospital

Gwardamangia

Malta

Tel. 0406-312015 Telex 406 HIEDUC

Söderman, Päivi

Dept. of Pediatrics

Östersunds Sjurhus

83200 Östersund

Sweden

Tel. 0046-63-153000 Fax 0046-63-153513

Thomas, Molly

Head of Clinical Pharmacology

Christian Medical College \& Hospital
IDA Scudder Road - Post Box 3

Vellore 632004

India

Tel. 0091-416-22603 Fax 0091-416-25035

Uppal, Roma

Dept. of Pharmacology

Post Graduate Institute

Medical Education and Research

160031 Chandigarh

India

Tel. 0091-32351 ext 323 Fax 0091-416-25035

Weerasinghe, Tilak

Dept. of Pharmacology

University of Colombo

P.O. Box 271 Kynsey Road

Colombo 8

Sri Lanka

Tel. 0094-1-636337

Yoshioka, Hajime

Dept. of Pediatrics

Asahikawa Medical College

Nishikagura 4-5-3-11

Asahikawa 078

Japan

Tel. 0081-166-652111 Telex 922492

\section{Dukes, M.N. Graham}

Professor of Drug Policy Science

University of Groningen

Frödings Alle 10

2860 Söborg

Denmark

Tel. 0045-39-661136 Fax 0045-39-660032

Lunde, Inga

Pharmaceuticals Programme

WHO EURO

8, Scherfigsvej

2100 Copenhagen

Denmark

Tel. 0045-31-290111 Fax 0045-31-181120

\section{Advisory Board}

Lunde, Per Knut

Dept. of Pharmacotherapeutics

Ullevâl University Hospital

University of Oslo

P.O. Box 1065

Blindern, N-OSLO 3 Norway

Tel. 0047-2-119005 Fax 0047-2-119013

Wagner, Marsden

Regional Officer for Maternal and Child Health - WHO-EURO

8, Scherfigsvej

2100 Copenhagen

Denmark

Tel. 0045-31-290111 Fax 0045-31-181120 